\title{
Nicht immer ist der bequemste Weg der beste, er kann auch ganz schön gefährlich sein
}

Ulrich Nägeli

Ich erlaube mir, wie Franz Marty, vorwiegend die männliche Form Arzt und Patient zu verwenden und möchte mich bei den Frauen für diese Unfairness aus Bequemlichkeit entschuldigen.

Korrespondenz: Dr. med. Ulrich Nägeli

Käsernstrasse 3

CH-8865 Bilten

Tel. 0556196020

ulrich.r.naegeli[at]hin.ch
Für einmal bin ich mit Franz Marty nicht einig. Sein Plädoyer für den Tiers payant (TP) ist ein Plädoyer für die Bequemlichkeit, es blendet leider wichtige grundsätzliche Aspekte weitgehend aus. Im Vorspann erwähnt Marty zwar wichtige Gebiete wie die ärzteeigene Datenbasis und technische Aspekte des Abrechnens, geht aber nicht auf Grundsätzliches der Patienten-Arzt-Beziehung ein und gar nicht auf die Verhältnisse im Dreieck Patient-Arzt-Versicherung.

Ein Punkt fehlt ganz: die Rechte der Patienten. Klar formuliert: Die Wünsche der Versicherungen sind nicht generell mit den Interessen der Patienten gleichzusetzen, obwohl das immer wieder und immer mehr so dargestellt wird. Die Wahrnehmung der Interessen ihrer Versicherten ist eine wichtige Aufgabe, darf aber nicht zum Blankoscheck werden. Im Vordergrund steht immer noch der Vertrag zwischen Hilfesuchenden und Behandelnden. Der haben den TP dazu instrumentalisieren wollen, die Macht über möglichst alle Daten zu erlangen. So wurden die Kantone Glarus und St. Gallen bei der TARMED-Einführung regelrecht geknebelt. In beiderseitigem Einvernehmen waren die Ostschweizer Ärztegesellschaften St.Gallen, Thurgau, Appenzell, Glarus und Schaffhausen und der (ausser Zürich) deckungsgleiche Regionalverband von santésuisse übereingekommen, den Tarif nicht kantonal, sondern regional umzusetzen. St. Gallen und Glarus wollten bei dieser Gelegenheit vom TP zum TG wechseln, sich also dem Modus der andern (AI/AR, TG und SH) anschliessen. Das wurde von den Versicherern verhindert, die Beibehaltung des TP zur conditio sine qua non für die Bildung der gemeinsamen Tarifregion erklärt. Die Absicht war klar: Im TP sind die Zahlen schneller bei den Versicherern. Sie wollten von den zwei Kantonen möglichst rasch Trendzah-

\section{Beim Tiers garant merkt man viel rascher, wenn jemand in finanziellen Schwierigkeiten ist.}

«Tiers» soll würdig, aber möglichst diskret, seine wichtige Aufgabe wahrnehmen. Das sieht auch das KVG so. Marty erwähnt das samt gesetzlicher Grundlage unter «Soll-Zustand», geht aber kaum darauf ein.

\section{Warum flammt dieser Dauerbrenner immer wieder auf?}

\section{Vorgeschichte}

Ich gehe nur auf die Entwicklung seit den Arbeiten am neuen KVG 1994 und seinem Inkrafttreten 1996 ein. Frühere Probleme um TP und TG (Tiers garant) sind wichtig, würden aber den Rahmen dieses Artikels sprengen.

Im Lauf der Diskussion um das neue KVG und die Einführung des TARMED kam in fast allen Kantonen, die noch im TP abrechneten, die Mehrheit der Ärzte zur Ansicht, der Wechsel zum TG sei ratsam. Die Krankenversicherer hatten zu dieser Zeit sehr viel Energie investiert, eine möglichst weitgehende Kontrolle über das System zu erlangen. Sie len. Die Steuerungsgruppe Ostschweiz sah sich 2004 dann in der schwierigen Lage, Zahlen aus zwei verschiedenen Modi zu bearbeiten. Sozusagen aus drei Äpfeln und zwei Birnen einen Most zu pressen, der allen Beteiligten möglichst gut mundete.

Nach Ablauf der Kostenneutralitätsphase wurden die Verträge in der ganzen Schweiz gekündigt. Beim Neuabschluss 2006 wechselten dann Glarus und St. Gallen wie fast alle andern Kantone zum TG.

Kaum waren die Verträge unterzeichnet, begann ein wahres Trommelfeuer der Versicherer auf die Ärzte, wieder zum TP zurückzukehren. Es ist also nicht so, wie Marty schreibt, dass sich der TG nicht durchgesetzt habe, er wurde vielmehr sofort auf massive Art untergraben. Es ist eher erstaunlich, dass so viele Kolleginnen und Kollegen trotz aller Schalmeienklänge und Druckversuche beim TG geblieben sind!

\section{Weitere Entwicklung}

Der Autor schreibt zu Recht, dass es der TG in Zukunft schwer haben werde. Dies aber in erster Linie, 
weil man ihn weiterhin aktiv schlecht macht und gegen ihn arbeitet. Die Krankenversicherer arbeiten hier klar entgegen dem Willen des Gesetzgebers; sie interpretieren die «kann»-Formulierung des KVG als Freipass, systematisch das Gegenteil zu tun. Entsprechend werden Vorteile des TP gebetmühlenartig wiederholt, Nachteile totgeschwiegen und umgekehrt. Ein Trend zurück zum TP wurde in den Kantonen, die gewechselt hatten, rasch spürbar. Gerade in einem kleinen Kanton erfährt man durch Patienten rasch, was sich tut: «Bei meinem Bruder geht das aber einfacher als bei Ihnen, sein Arzt schickt die Rechnung direkt der Kasse». Damit das Thema offen angegangen werde, habe ich 2009 an der Mitgliederversammlung der Glarner Ärztegesellschaft Antrag gestellt, der Vorstand solle beauftragt werden abzuklären, wie weit die Beschlüsse eingehalten würden oder umgekehrt, ob der Eindruck stimme, sie würden immer mehr unterlaufen.

Unser Vorstand hat ganze Arbeit geleistet, die Situation wurde abgeklärt, und wir haben an zwei Mitgliederversammlungen offen darüber gesprochen. Die «Abtrünnigen» sind es zwar geblieben, die «Starrköpfigen» haben die Gründe erfahren und für einzelne Situationen auch Verständnis.

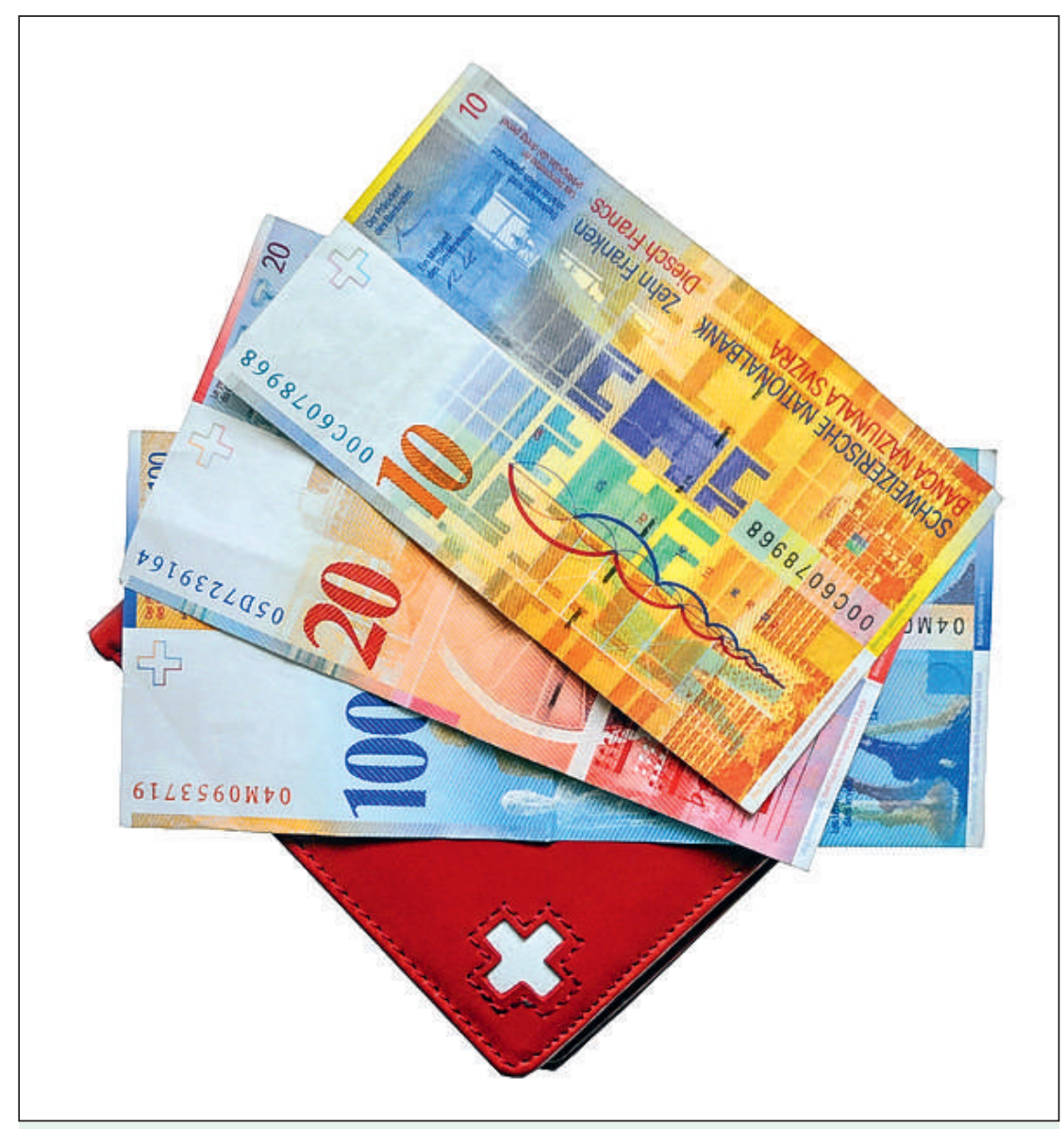

Der Tiers garant macht den Patienten sehr deutlich, welche Kosten ihre Behandlung verursacht.

\section{Zu den Vor- und Nachteilen beider Systeme}

Aufgeführt werden im Artikel vorwiegend die Vorteile des TP und die Nachteile des TG. Sichten wir also zuerst einmal die Liste:

- Leute mit kleinem Geldbeutel seien im TG finanziell oft überfordert: Das ist grundsätzlich so nicht wahr. Sie sind es oft, einverstanden, mit der Zahlungsabwicklung. Das sind sie aber auch in andern Bereichen, wie ich aus der Tätigkeit in der Sozialbehörde weiss. Wohnungsmiete und allgemeiner Lebensunterhalt sind die grossen Kostenbrocken, dazu kommt meist das Auto, fast ausnahmslos ein Mobiltelefon und E-Kommunikation, Freizeit, Ferien, Zahnarzt und vieles mehr. Im Gegensatz zu den Gesundheitskosten sind diese Bereiche nicht durch Prämienverbilligung und maximale Kostenbeteiligung abgefedert. Wir tun gerade diesen Leuten keinen Gefallen, wenn wir die Bezahlung der ärztlichen Grundversorgung an ihnen vorbeischleusen, ohne sie wissen zu lassen, welche Kosten entstehen. Es ist blauäugig zu glauben, sie nähmen die Beträge wirklich wahr, wenn die Zahlungen direkt abgewickelt werden, selbst wenn sie Rechnungskopien erhalten.

- Es bestehe Gefahr, dass ein beträchtlicher Teil der Bevölkerung ausgegrenzt werde oder nur mit Mühe adäquate medizinische Betreuung finde: ebenso falsch! Wenn die, im Gesamtbudget meist eher geringen, Beträge wirklich zum Problem werden, bestehen Möglichkeiten, Hilfe zu erhalten. Dafür gibt es die Sozialbehörde. Ich empfinde es auch als ärztliche Aufgabe, sie darauf aufmerksam zu machen und ihnen allenfalls zu helfen, diesen Weg zu finden und zu gehen. Mit dem Wechsel vom TP zum TG habe ich eines festgestellt: Beim TG merkt man viel rascher, wenn jemand in finanziellen Schwierigkeiten ist, im TP oft erst, wenn die Krankenkasse eine Leistungssperre verhängt.

- In Kantonen mit freier Wahl des Medikamentenbezugs verschärfe sich die Problematik: Das mag stimmen, hier muss ich anmerken, dass der Patient auf jeden Fall das Recht hat zu entscheiden, ob er Medikamente direkt beim Arzt oder in der Apotheke beziehen will. Für den Arzt kann bei schlechten Zahlern die Aufteilung zwischen Arztkosten und Medikamenten sogar ein Vorteil sein.

- Der Aufwand für das Mahnwesen werde im TG immer bedeutend grösser sein als im TP: Der Aufwand ist effektiv grösser, aber das Ergebnis sicherer. Ich habe erwähnt, dass man im TG rasch merkt, wenn Probleme auftreten, nämlich sobald Rechnungen nicht bezahlt werden. Im TP geht das länger; man wähnt sich in Sicherheit, bis plötzlich die Versicherung unsere Leistungen nicht vergütet. Leistungssperre! Ab diesem Punkt wird es sehr mühsam, denn die Bezahlung er- 
folgt erst, wenn der Schuldner seinen Verpflichtungen gegenüber der Kasse nachgekommen ist. Dies kann lange dauern, man hat kaum Möglichkeiten, hier beschleunigend einzugreifen. Die Versicherer sind nicht sehr mitteilsam, pochen auf den Datenschutz, teilen meist auch nicht mit, wenn die Sperre aufgehoben wird. Möglich, aber mühsam ist der Gang über die Sozialbehörde. Unsere persönliche Erfahrung ist, dass der Aufwand seit dem Wechsel zum TG kaum grösser geworden ist, die Erfolgsraten aber markant gestiegen sind. Wir haben fast nur noch Ausstände aus dem Notfalldienst.

\section{Kostenwahrheit bei uns, Verantwortung und}

Kostenbewusstsein bei den Versicherten.
Franz Marty schreibt, die Argumente gegen den TP überzeugten wenig, er führt deren zwei auf:

- Rechnungskontrolle durch den Patienten: In einem Punkt stimme ich zu: TARMED-Rechungen sind für Nichtfachleute kaum lesbar. Dann sollte man aber hier ansetzen und nicht das Prinzip der Rechnungskontrolle abschaffen. Die Möglichkeit, sie auch im TP zu bieten, besteht, wird aber erfahrungsgemäss wenig genutzt.

- Kostenwahrheit: Wenn wir in diesem Bereich bald noch die Einzigen sind, die das Prinzip hochhalten, ist das für mich kein Argument, es abzuschaffen. Wir sollten eher darauf hinarbeiten, dass es andere auch tun! Die Leute sollten merken, dass sie z.B. in unserem Kanton aufgrund der unterschiedlichen Taxpunktwerte 20\% höhere Kosten verursachen, wenn sie direkt das Spital aufsuchen. Noch besser wäre es, wenn sie die Differenz selber bezahlen müssten, doch das ist eine andere Diskussion.

- Die Einbindung des TG in den elektronischen Verarbeitungsprozess sei mühsam und in $\mathrm{Zu}$ kunft vermutlich das grösste Hindernis für den Erfolg des TG: Das erstaunt mich. Ich bin perplex, da ich den Autor als Pionier im Bereich von eHealth kenne und schätze! Abrechnungen jeder Art sind seit jeher die Domäne des elektronischen Datenverkehrs, in sozusagen allen Lebensbereichen. Es gibt Lösungen für fast alles, die Systeme kommen mit Rückzahlungen, Rabatten, Zahlungsaufteilungen, Mahnwesen und vielem anderen zurecht. Ausgerechnet bei eHealth soll das nun zum Problem werden?

Die Vorteile des TP werden geflissentlich aufgelistet. Sie sind durchaus vorhanden, aber vorwiegend im Bereich der Bequemlichkeit anzusiedeln:

- Der Hauptvorteil des TP liege in einer markanten Verminderung des Debitorenaufwandes. Stimmt, er ist gestiegen, als wir von der Sammelrechnung an die Versicherer zu Einzelrechnungen gewechselt haben. Ich schätze aber, er ist etwa gleich, wenn man der Verpflichtung nachkommt, dem Patienten eine Rechnungskopie zuzustellen.

- Die bessere Integration in den eHealth-Workflow wage ich zu bezweifeln. Noch einfacher wäre es tatsächlich, wenn die Krankenversicherer die elektronische Rechnungstellung via Trust-Center endlich akzeptieren würden. Dass sie es nicht tun, ist nicht ein Defekt des TG, sondern klares Kalkül, die Trust-Center auszuschalten und alleinige Datenhoheit zu erlangen.

kommentien? diesen Artike Sie dafür die Kommentarfunktion in der OnlineVersion oder sehen Sie nach, was Ihre Kolleginnen und Kollegen bereits geschrieben haben: www.saez.ch/ aktuelle-ausgabe/ interaktive-beitraege/
- Der TP ermögliche auch in Zukunft allen Bevölkerungsgruppen fairen Zugang zur Primärversorgung: der TG auch, ich sehe wirklich keinen Unterschied. Wo es echte Probleme gibt, braucht es andere Lösungsansätze, beispielsweise den Einbezug eines Sozialarbeiters oder Hilfe durch Organisationen wie Pro Senectute.

\section{Schlussfolgerungen}

Franz Marty hat vielleicht recht, dass der damalige Wechsel zum TG in den meisten Gebieten, die ihn nicht bereits anwendeten, wenig gebracht habe. Jedenfalls nicht viel direkt Sichtbares, möchte ich eher sagen. Die Ärzteschaft versuchte damals ein Zeichen zu setzen, der Autor anerkennt dies und weist selber auf den Aufbau wertvoller Strukturen hin, der damit verbunden war. Er spricht dann vom «Beharren» auf dem TG. Diese Wortwahl spiegelt wider, dass der TG eben Gegner hat, denen er ein Dorn im Auge ist. Schon kurz nach seiner Einführung sind sie auf fast unanständige Art aktiv geworden. Sie haben leider inzwischen Erfolge erzielt, die zuerst solide geglaubte Basis ist mancherorts recht unterspült worden. Der Wechsel zurück zum TP ist meist aus Gründen der Bequemlichkeit erfolgt. Dazu steht man besser, als nun hehre Gründe wie «den besseren Zugang zum Gesundheitssystem für finanzschwache Patienten» zu bemühen. Letztlich bleibt es ein Nullsummenspiel, es fliessen die gleichen Beträge, im TP wie im TG. Bleiben wir doch lieber den Werten treu: Kostenwahrheit bei uns, Verantwortung und Kostenbewusstsein bei den Versicherten, möglichst direktes Verhältnis zwischen Patient und Arzt auch in diesem Bereich.

Ein Rest an Selbständigkeit in der Berufsausübung sollte uns auch etwas wert sein; wir werden immer mehr gegängelt und lassen uns das mit immer weniger Widerstand bieten. Ich ertappe mich manchmal dabei, nach rechts und links zu schielen im näheren beruflichen Umfeld, beispielsweise den Zahnärzten und Veterinären, und wundere mich dann doch etwas über meine eigene Situation.

1 Marty F. Stürzt der Tiers payant die Ärzte ins Verderben? Schweiz Ärztezeitung. 2014;95(18):710-2. 